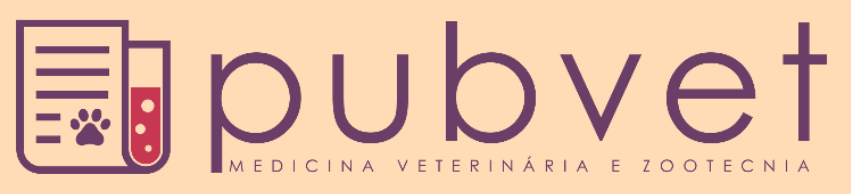

https://doi.org/10.31533/pubvet.v12n11a203.1-5

\title{
Diversidade da entomofauna em um plantio de cacau (Theobroma cacao) no município de Ilhéus, Bahia, Brasil
}

\author{
Marlon dos Santos Pereira Birindiba Garuzzo ${ }^{*} \bullet$, Rosane Rodrigues da Costa Pereira $^{2} \bullet$ \\ ${ }^{1}$ Acadêmico de B.I em Ciências, Universidade Federal do Sul da Bahia, 45604-811, Ilhéus-BA, Brasil. \\ ${ }^{2}$ Engenheira Agrônoma, Dr., Professora do Centro de Formação em Ciências e Tecnologias Agroflorestais, Universidade \\ Federal do Sul da Bahia, 45604-811, Ilhéus-BA, Brasil. \\ *Autor para correspondência, E-mail: marlongaruzzo@gmail.com
}

\begin{abstract}
RESUMO. O cacau (Theobroma cacao) é a principal cultura de interesse econômico na região do Sul da Bahia. Em decorrência da crise de 1990, causada pelo fungo (Moniliophthora perniciosa), as lavouras foram devastadas, transformando totalmente o ambiente antes equilibrado. $\mathrm{O}$ objetivo deste trabalho foi analisar a entomofauna em um plantio de cacau em Ilhéus-BA, território afetado pelo patógeno, e verificar a riqueza e abundância dos insetos. Foi realizada coleta, por meio de armadilha tipo "Pitfall" de insetos na área, os identificando em nível de família e posteriormente a avaliação da diversidade. Foram encontrados insetos pertencentes a cinco ordens, sendo as mais abundantes, Hymenoptera $(78,6 \%)$ e Coleoptera $(15,7 \%)$. A família Formicidae, da ordem Hymenoptera, foi mais encontrada em pontos de coleta, e a ordem Coleoptera, apresentou maior número de famílias. A diversidade de insetos medido pelo índice de Shannon Wienner (SW), adaptado para a classificação de famílias é de 0,98.
\end{abstract}

Palavras chave: Diversidade, insetos, cacau

\section{Diversity of the entomofauna in a cocoa plantation (Theobroma cacao) in the municipality of Ilhéus, Bahia, Brazil}

\begin{abstract}
ABSTRAC. The cocoa (Theobroma cacao) is the culture of economic interest in the southern region of Bahia. As a result of the 1990 crisis, caused by the fungus (Moniliophthora perniciosa), the crops were devastated, totally transforming the previously balanced environment. The objective of this work was to analyze the entomofauna in a cocoa plantation in Ilhéus, Bahia, territory affected by the pathogen, and verify the richness and abundance of the insects. A Pitfal trap of insects was collected in the area, identifying them at the family level and then evaluating the diversity. Insects belonging to five orders were found, with the most abundant being Hymenoptera (78.6\%) and Coleoptera (15. 7\%).The Formicidae family, of the order Hymenoptera, was more found in collection points, and a Coleoptera order, in a greater number of families. The diversity of insects measured by the Shannon Wienner index (SW), adapted for the family classification is 0.98 .
\end{abstract}

Keywords: Diversity, insects, cocoa

\section{Diversidad de la entomofauna en cultivo de cacao (Theobroma cacao) em el municipio de Ilhéus, Bahia, Brasil}

RESUMEN. El cacao (Theobroma cacao) es el principal cultivo de interés económico en la región sur de Bahía. Con relación a la crisis de 1990, por la fase del hongo (Moniliophthora perniciosa), los cultivos fueron devastados, transformando totalmente el 
ambiente antes equilibrado. El objetivo de este trabajo fue analizar la entomofauna en una plantación de cacao en Ilhéus-BA, territorio afectado por el patógeno, verificar la riqueza y la presencia de los insectos. La colecta fue realizada usando armadilla tipo "Pitfall" de insectos en el área., identificándolos a nivel de familia y posteriormente la avaluación de la diversidad. Se encontraron insectos pertenecientes a cinco órdenes, siendo los más abundantes, Hymenoptera $(78,6 \%)$ y coleópteros $(15,7 \%)$. La familia Formicidae, del orden Hymenoptera, fue más encontrada en puntos de colecta, y el orden Coleoptera, en mayor número de familias. La diversidad de insectos medidos por el índice de Shannon Wienner (SW), adaptado para la clasificación de familias es de 0,98.

Palabras clave: Diversidad, insectos, cacao

\section{Introdução}

A fauna existente no solo da serapilheira tem a característica de alta diversidade e rápida capacidade de reproduzir, sendo considerados exímios bio-indicadores e suas propriedades e funções podem indicar e determinar a qualidade do solo. Esses fatores podem ser avaliados pela presença de organismos específicos, análise da comunidade e processos biológicos como, a modificação estrutural do solo e sua decomposição. Por isso, os insetos edáficos que são o conjunto de insetos terrestres que dependem diretamente do solo e auxiliam a alimentação dos organismos decompositores são fatores determinantes, pois as decomposições da serapilheira aceleram os níveis de ciclagem de nutrientes indicando a qualidade do solo (Knoepp et al., 2000; Wink et al., 2005).

O cacau (Theobroma cacao) é originário das regiões pluviais da América Tropical, onde até hoje pode ser encontrado em seu estado silvestre, é uma planta perene, com florações no verão e frutificação no outono e inverno, propaga-se por sementes e tem preferência edafoclimáticas o clima quente e úmido e os solos arenosos (Sánchez, 2011). No Brasil o cultivo adaptou-se principalmente às condições climáticas e ao Bioma Mata Atlântica na região no sul Baiano, onde foi introduzido no ano de 1746 com o cultivo do tipo Forasteiro, variedade mais difundida, atualmente com cerca de $80 \%$ da produção mundial. Mas somente em 1752 teve-se a primeira produção cacaueira no município de Ilhéus (Fausto \& Fausto, 1994).

O cacau é a principal cultura de interesse econômico na região litoral sul da Bahia (CEPLAC, 2013; Sodré, 2017). Nos últimos tempos, a região sofreu com uma mudança muito importante no uso de seus solos, em decorrência da crise de 1990, quando as lavouras sofreram com a doença "vassoura de bruxa" causada pelo fungo (Moniliophthora perniciosa) devastando lavouras e causando enorme prejuízo aos cacauicultores (CEPLAC, 2013; Fausto \& Fausto, 1994).

O manejo intensivo do solo, a aplicação de produtos fitossanitários e fertilizantes tornaram-se práticas comuns contra o patógeno da "vassoura de bruxa", que chega a causar perdas de até $90 \%$ da produção (Sodré, 2017). O uso destas práticas antrópicas pode ocasionar perda de matéria orgânica, alterações no ecossistema e da biodiversidade existente no solo, contaminação das águas subterrâneas, erosão e danos aos processos bioquímicos (Araújo \& Monteiro, 2007; Arshad \& Martin, 2002).

A riqueza taxonômica dos insetos é muito grande e sua a distribuição no espaço é irregular em sua maioria, sendo impossível inventariar todos os indivíduos, mesmo para uma área pequena. Assim extrapolações são necessárias para a realização da riqueza total. O levantamento de insetos, depende de cálculos para estimar a diversidade taxonômica e identificação do número dos insetos de cada grupo de artrópodes, para descobrir seus efeitos no ecossistema (Gullan et al., 2007).

Este estudo foi realizado para analisar a entomofauna em um plantio de cacau no município de Ilhéus-BA, verificando a riqueza e abundância das ordens dos insetos, e sua variedade de famílias.

\section{Material e métodos}

A área de estudo é um plantio de cacau com sistema de cultivo consorciado com pupunha e seringueira, localizado no perímetro rural do município de Ilhéus-BA $\left(14^{\circ} 45\right.$ '53.62"S, $39^{\circ} 14^{\prime} 9.95^{\prime \prime O}$ ) (Figura 1). A captura dos insetos foi realizada com armadilha "pitfall", constituída de um recipiente coletor de plástico com capacidade para $1 \mathrm{~L}$, enterrada até o nível do solo e preparada com $1 \mathrm{ml}$ de sabão líquido para quebrar a tensão superficial da água e $400 \mathrm{ml}$ de água, sem mecanismo de atração. 

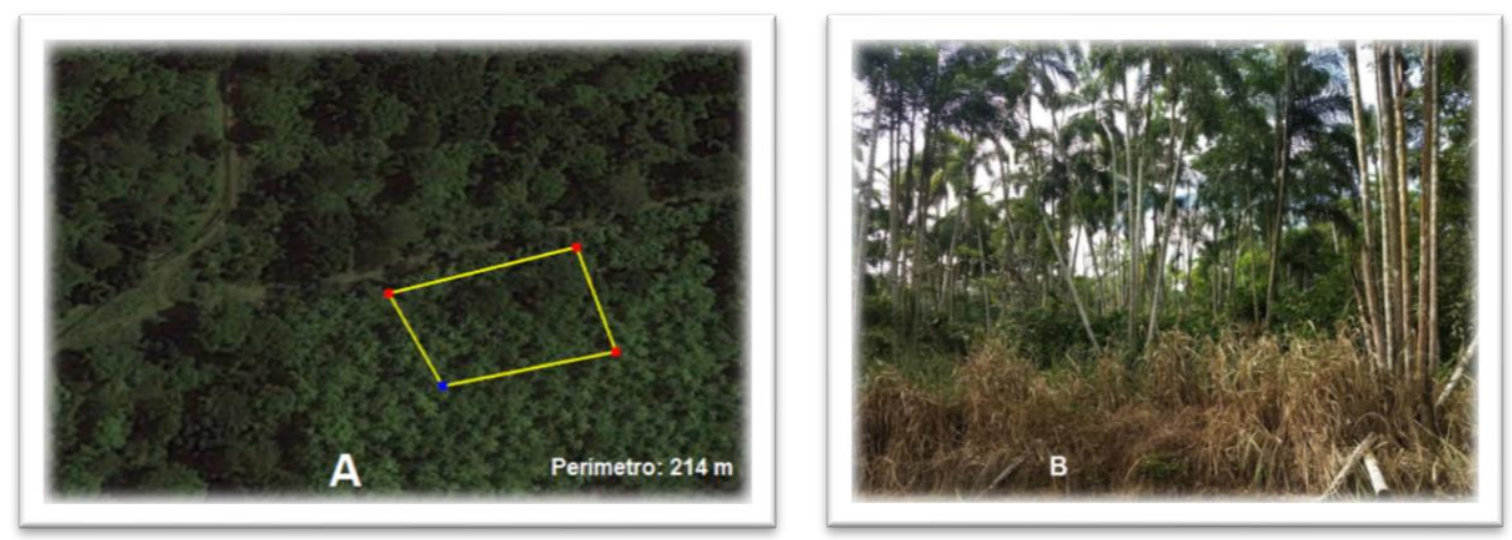

Figura 1. (A) Localização da área de estudo (Fonte: Google Earth Pro). (B) Lateral da área do plantio de cacau com cultivo consorciado.

Os insetos coletados foram conservados em álcool $70 \%$ e levados para o laboratório do Centro de Formação em Ciências e Tecnologias Agroflorestais para contagem, triagem e identificação em nível de família, por meio de chaves de taxonômicas de dupla entrada com auxílio de um microscópio estereoscópio e posteriormente será depositado na coleção entomológica da UFSB - Universidade Federal do Sul da Bahia.

A análise de diversidade de insetos encontrados foi realizada por meio do índice de Shannon Wiener, adaptado para família, este serve como indicador do equilíbrio do sistema ecológico e leva em conta a uniformidade das abundâncias de espécies para saber a qual espécie pertenceria um indivíduo retirado aleatoriamente da população, usando o programa BioEstat (versão 5.3). A abundância $(\mathrm{N})$ representa o número de indivíduos coletados na "Pitfall" e a análise da frequência foi calculada com base na quantidade de amostras em que o táxon ocorreu, multiplicado por 100 , sendo a família mais constante quando atingiu uma frequência $\mathrm{F} \geq 50 \%$, comum quando presente entre $10 \%$ e $49 \%$ e rara quando ocorreu em $\mathrm{F}<10 \%$.

\section{Resultados e discussão}

Foram capturados 140 exemplares de insetos distribuídos em oito ordens, sendo Hymenoptera a mais representativa, com $\mathrm{N}=110(78,6 \%)$, seguida de Coleóptera $\mathrm{N}=22$ (15,71\%), Orthoptera $\mathrm{N}=4(2,85)$, Dermaptera $\mathrm{N}=2$ $(1,42 \%)$, Isoptera $\mathrm{N}=1(0,71 \%)$ e Díptera $\mathrm{N}=1$ $(0,71 \%)$, e 11 famílias, ressaltando-se a Formicidae (Hymenoptera) com $\mathrm{N}=11(78,6 \%)$, Carabidae (Coleoptera) $\mathrm{N}=5(3,58 \%)$ e Erotylidae (Coleoptera) $\mathrm{N}=5(3,58 \%)$ (Tabela 1).
Tabela 1. Abundância $(\mathrm{N})$ e frequência relativa $(\%)$ de insetos capturados em abril de 2018 no plantio de cacau no município de Ilhéus, BA.

\begin{tabular}{lcc}
\hline Táxon & Abundância (N) & Frequência relativa (\%) \\
\hline Hymenoptera & 110 & 78,6 \\
Formicidae & 110 & 78,6 \\
Diptera & 1 & 0,71 \\
Muscidae & 1 & 0,71 \\
Dermaptera & 2 & 1,42 \\
Chelisochidae & 1 & 0,71 \\
Spongiphoridae & 1 & 0,71 \\
Isoptera & 1 & 0,71 \\
Kalotermitidae & 1 & 0,71 \\
Coleoptera & 22 & 15,71 \\
Staphylinidae & 4 & 2,85 \\
Caracbidea & 5 & 3,58 \\
Erotylidae & 5 & 3,58 \\
Chrysomelidae & 4 & 2,85 \\
Coccinellidae & 4 & 2,85 \\
Orthoptera & 4 & 2,85 \\
Gryllidae & 4 & 2,85 \\
\hline Total & 140 & 100 \\
\hline
\end{tabular}

Entre os insetos coletados foi verificado a presença de inimigos naturais, predadores que se alimentam de outros insetos, exemplares são as famílias Chelisochidae e Sponghiphoroidea, da ordem Dermaptera, mais conhecida como tesourinhas, importantes predadoras de ovos de pulgões, moscas brancas, lagartas brancas e pupas em geral, durante o estágio de larva e adultos. As famílias Carabidea e Coccinellidae, da ordem Coleoptera, que em fase larval e adulta também se alimentam de insetos, como pulgões, cochonilhas, ácaros e larvas. Estes insetos são importantes para o controle biológico de pragas na lavoura, evitando a superpopulação de insetos que podem vir a causar injuria a lavoura e consequentemente danos econômicos ao produtor (Silva et al., 2013). 
Nas ordens Hymenoptera, Orthoptera, Isoptera e Diptera somente foi encontrado uma única família, mas o número de indivíduos da Família Formicidae é extremamente maior comparado as outras famílias identificadas. As formigas são um grupo de insetos sociais, capazes de habitar os mais variados ambientes, tendo sua maior biodiversidade nas regiões tropicais. As espécies de Formicidae dentro das comunidades são influenciadas pela distribuição dos recursos disponíveis, assim como sua estratégia de obtenção (Fowler et al., 1991).

Os hábitos alimentares são bastante variáveis, existindo onívoras, que são generalistas, podendo explorar mais recursos, exemplo são espécies do gênero Camponotus, Pheidole e Solenopsis; predadoras, como a espécie Neivamyrmex pilosus e do gênero Hypoponera; as que se alimentam por líquidos, como a Linepithema sp.; e fungívoras, como a Atta sp. e Mycocepurus goeldii (Battirola et al., 2005). As famílias encontradas no estudo se analisadas a nível de espécies, possivelmente em maioria serão terrícolas e que nidificam principalmente no solo, ou seja, aquelas que geralmente estão associadas a fragmentos de decomposição de folhas, galhos ou madeira caída. É possível que as formigas sejam capazes de transportar organismos patogênicos, assim na lavoura de cacau, esse grande índice de indivíduos pode ter sido um fator de rápida propagação dos fungos Moniliophthora perniosa, causador das injurias no cacau, na crise da década de 90 (Fonseca et al., 2010). A diversidade de insetos encontrados, medido pelo índice de Shannon Wiener (SW) e levando em consideração a adaptação para análise de famílias é de 0,98, resultado relativamente baixo, mas é importante notar que a classificação quanto a família é mais ampla e abrangente do que a de espécie.

\section{Conclusão}

As ordens Hymnoptera e Coleoptera apresentaram maior abundância de insetos. Todavia, é verificado maior número de indivíduos na Hymenoptera e mais diversidade de famílias na Coleoptera. É significativo para a lavoura de cacau a presença das famílias Chelisochidae, Sponghiphoroidea, Carabidea, Coccinellidae, controladores biológicos de insetos pragas.

Recomenda-se estudo da Família Formicidae e sua possível relação como fator para a propagação do fungo Moniliophthora perniosa.
O estimador de diversidade aponta para a possiblidade de existir muitas espécies na área, em um período maior de coleta.

\section{Referências}

Araújo, A. S. F., \& Monteiro, R. T. R. 2007. Indicadores biológicos de qualidade do solo. Bioscience Journal, 23(3), 66-75.

Arshad, M. A., \& Martin, S. 2002. Identifying critical limits for soil quality indicators in agroecosystems. Agriculture, Ecosystems \& Environment, 88(2), 153-160.

Battirola, L. D., Marques, M. I., Adis, J., \& Delabie, J. H. C. 2005. Composição da comunidade de Formicidae (Insecta, Hymenoptera) em copas de Attalea phalerata Mart.(Arecaceae), no Pantanal de Poconé, Mato Grosso, Brasil. Revista Brasileira de Entomologia, 49(1), 107-117.

CEPLAC. 2013. Cacaueira-ceplac (Vol. 10). Ilhéus, Bahia, Brasil.

Fausto, B., \& Fausto, S. 1994. História do cacau no Brasil (Vol. 1). São Paulo, São Paulo, Brasil: Edusp.

Fonseca, A. R., Batista, D. R., Amaral, D. P., Campos, R. B. F., \& Silva, C. G. 2010. Formigas (Hymenoptera: Formicidae) urbanas em um hospital no município de Luz, Estado de Minas Gerais. Acta Scientiarum. Health Sciences, 32(1), 107-117.

Fowler, H. G., Forti, L. C., Brandão, C. R. F., Delabie, J. H. C., \& Vasconcelos, H. L. 1991. Ecologia nutricional de formigas. In A. R. Panizzi \& J. R. P. Parra (Eds.). Ecologia nutricional de insetos e suas implicações no manejo de pragas (pp. 131-223). São Paulo, São Pauoo, Brasil: Editora Manole Ltda.

Gullan, P. J., Cranston, P. S., McInnes, K. H., \& Hoenen, S. M. M. 2007. Os insetos: um resumo de entomologia. São Paulo, São Paulo, Brasil: Roca.

Knoepp, J. D., Coleman, D. C., Crossley Júnior, D. A., \& Clark, J. S. 2000. Biological indices of soil quality: an ecosystem case study of their use. Forest Ecology and Management, 138(13), 357-368.

Sánchez, S. E. M. 2011. Cacau e Graviola: Descrição e danos das principais pragas-deInsetos (Vol. 1). Ilhéus, Bahia, Brasil: Editus. 
Silva, A. C. R. A., Gomes, C. C., Sacramento, F. Z., Garcia, G. L., Schultz, H., Pian, L. B., Tamashiro, L. A. G. 2013. Guia para o reconhecimento de inimigos naturais de pragas agrícolas. Brasília, Brasil.

Sodré, G. A. 2017. Cultivo do cacaueiro no estado da Bahia. São Félix do Xingu, Pará, Brasil: Associação para o desenvolvimento da agricultura familiar do alto xingu - ADAFAX.

Wink, C., Guedes, J. V. C., Fagundes, C. K., \& Rovedder, A. P. 2005. Insetos edáficos como indicadores da qualidade ambiental. Revista de Ciências Agroveterinárias, 4(1), 60-71.

Recebido: 23 Maio 2018 .

Aprovado:20 Junho 2018

Publicado: 20 Novembro 2018.

Licenciamento: Este artigo é publicado na modalidade Acesso Aberto sob a licença Creative Commons Atribuição 4.0 (CC-BY 4.0), a qual permite uso irrestrito, distribuição, reprodução em qualquer meio, desde que o autor e a fonte sejam devidamente creditados. 